\title{
The Complex Amorphous Phase in Poly(butylene succinate-ran-butylene azelate) Isodimorphic Copolyesters
}

Idoia Arandia ${ }^{1}$, Agurtzane Mugical ${ }^{1}$, Manuela Zubitur ${ }^{2}$, Rosica Mincheva ${ }^{3}$, Philippe Dubois ${ }^{3,4}$, Alejandro J. Müller ${ }^{* 1,5}$ and Angel Alegría ${ }^{* 6}$

${ }^{1}$ POLYMAT and Polymer Science and Technology Department, Faculty of Chemistry, University of the Basque Country UPV/EHU, Paseo Manuel de Lardizabal 3, 20018 Donostia-San Sebastián, Spain

${ }^{2}$ Chemical and Environmental Engineering Department, Polytechnic School, University of the Basque Country UPV/EHU, 2008 Donostia-San Sebastián, Spain

${ }^{3}$ Laboratory of Polymeric and Composite Materials, Center of Innovation and Research in Materials and Polymers (CIRMAP), University of Mons (UMONS), Place du Parc 20, 7000, Mons, Belgium

${ }^{4}$ Materials Research and Technology Department (MRT), Luxembourg Institute of Science and Technology (LIST), Rue du Brill, 41, 4422 Belvaux, Luxembourg

${ }^{5}$ IKERBASQUE, Basque Foundation for Science, Bilbao, Spain

${ }^{6}$ Departamento de Física de Materiales, University of the Basque Country UPV/EHU and Centro de Física de Materiales (CFM) (CSIC-UPV/EHU) - Materials Physics Center (MPC), Paseo Manuel de Lardizabal 5, 20018 San Sebastián, Spain

*Corresponding authors 


\begin{abstract}
Poly(butylene succinate-ran-butylene azelate) random copolyesters (PBS-ran PBAz) were studied using Broadband Dielectric Spectroscopy (BDS) and Differential Scanning Calorimetry (DSC). These copolymers are characterized by being isodimorphic and by displaying a eutectic behavior. Depending on their composition, the PBS rich phase, the PBAz rich phase or both phases can crystallize. The complex development of the amorphous phase, especially at compositions around the eutectic point was demonstrated by BDS results. The comonomer fraction included in the amorphous phase was quantified from the relaxation strength of the dielectric $\beta$ relaxations and thus the crystalline fraction can be calculated. The good agreement between the values determined from BDS and the crystallinity obtained from DSC experiments demonstrate that the degree of comonomer exclusion in these random copolymers during crystallization is far larger than comonomer inclusion. However, the glass transition temperatures determined from the dielectric $\alpha$ relaxation behavior are lower in most of the copolymers than those measured by DSC, whereas they are about the same in the homopolymers. This result is probably caused by a significant amount of amorphous phase in the copolymers that is not influenced by crystallinity. Furthermore, the crystallization behavior of these copolymers at different cooling protocols showed that the $\alpha$ relaxation not only depends on the crystalline fraction but also on the details of the crystallization process in these complex multiphasic copolymers.
\end{abstract}

Keywords: Biobased copolyesters, isodimorphism, random copolymers, dielectric relaxation, amorphous phase, semicrystalline polymers, molecular motions. 


\section{INTRODUCTION}

Biodegradable polymers have attracted much attention as they are considered to cause less pollution problems as compared to conventional plastic waste. ${ }^{1}$ Aliphatic polyesters, ${ }^{2-4}$ such as poly(lactic acid) (PLA), poly( $\varepsilon$-caprolactone) (PCL), poly(butylene succinate) (PBS) and poly(butylene azelate) (PBAz), which may also be obtained from renewable sources, can be considered as green polymers.

PBS is an interesting material in view of its good mechanical properties, which are comparable with those from low-density polyethylene and other polyolefins, ${ }^{5,6}$ relatively low production cost and good thermal properties. Additionally, the monomers for its synthesis are derived from biomass. ${ }^{7,8}$ PBS is also characterized by its high crystallinity, a characteristic that limits its biodegradation rate. Therefore, in order to tailor its crystallinity the synthesis of random copolyesters with other bio-based comonomers has been done in order to obtain versatile random copolymers like poly(butylene succinate-ran-adipate) (PBSA), poly(butylene succinate-ran-ethylene succinate) (PBSES) and more recently poly(butylene succinate-ran-butylene azelate) (PBSAz). ${ }^{9-15}$ The azelaic acid is industrially produced by the ozonolysis of oleic acid. The latter and succinic acid have same functional groups and they differentiated only in the length of the corresponding dicarboxylic acid. The random copolymers composed by BS and BAz are able to crystallize in the entire composition range despite being random. ${ }^{12-14,16}$

Random copolymers constituted by two crystallizable units can display different crystallization behavior depending on the miscibility of the two comonomers and ability to share crystal lattices. ${ }^{17-19}$ Comonomer units can be totally excluded from the crystalline 
regions to the amorphous phase or cocrystallization may be observed. Two cases of cocrystallization behavior have been reported: in isomorphic polymers, only one crystalline phase containing both comonomer units is observed. In the case of isodimorphic polymers, two crystalline phases are observed depending on composition. In each phase one crystal structure predominates (i.e., the majority phase) with the inclusion of a certain amount of the minor comonomer. The increase of the minor comonomer concentration within each crystalline phase changes the crystallization and melting behavior of the copolymer.

In previous works, Díaz et al. ${ }^{12,13}$ studied the structure of poly(butylene succinateran-butylene azelate) copolymers by wide angle X-ray scattering (WAXS) concluding that comonomer exclusion was predominant during crystallization. Some of us have shown in a previous publication that these copolymers can be considered isodimorphic. ${ }^{14}$ WAXS results showed that small but reproducible changes were produced in the crystalline unit cell of the dominant crystalline phase indicating that at least some comonomer inclusion was present during non-isothermal crystallization supporting the hypothesis that isodimorphic behavior was observed for PBS-ran-PBAz copolymers. Furthermore, in a recent work $^{15}$ we have employed SSA (successive self-nucleation and annealing), a thermal fractionation technique that promotes segregation of molecular defects that interrupt crystallizable sequences, to fractionate PBS-ran-PBAz. This thermal treatment allows a more thorough examination of the isodimorphic nature of the copolymers, and small angle X-ray scattering (SAXS) and WAXS analysis of the samples before and after SSA fractionation concluded that the degree of comonomer exclusion remained almost unchanged, corroborating the isodimorphic behavior of the copolymers. 
The crystallinity of polymeric materials affects the properties of the remaining amorphous phase in a complex way. It has been generally found that the glass transition of semicrystalline polymers occurs at higher temperatures than that of the corresponding fully amorphous material, additionally, the glass transition range for these semicrystalline materials is usually much broader. ${ }^{20}$ These facts reflect constrains that the crystalline phase exert on the amorphous phase of polymers, which eventually lead to the disappearance of the glass transition for materials with crystallinity around $50 \%$ or higher. This result is usually attributed to the presence of amorphous segments forming what has been called the rigid amorphous fraction (RAF), ${ }^{20}$ whose motions are severely restricted by the crystallites. In this view, a semicrystalline polymer would be composed by at least 3 fractions: the crystalline one, the RAF and the (mobile) constrained amorphous fraction or CAF. The latter would be responsible for the measurable glass transition by DSC, whereas the melting process and the X-ray diffraction reflections are manifestations of the crystalline phase. The RAF still has local mobility, as proved by its contribution to the secondary relaxations. ${ }^{21}$ In addition, in the initial stages of crystallization, the experiments evidence the coexistence of these two amorphous fractions with the unconstrained amorphous fraction (UCAF) which is characteristic of the fully amorphous material. ${ }^{22,23}$

Taking all this into account, one could expect that the situation encountered in copolymers with crystallizable components is probably more intricate since each of the 3 amorphous fractions described above could be found, each one with a different comonomer concentration. In this work, we have investigated this complex case in poly(butylene succinate-ran-butylene azelate) isodimorphic copolymers. By analyzing the dielectric relaxation of these materials we have had access to both the local and segmental dynamics. The data analysis provided the distinct contributions of the succinate and azelate groups to 
the local copolymer relaxation allowing a quantification of the fraction of each component involved in the crystalline phase. On the other hand, the behavior of the dielectric relaxation loss peak above the glass transition temperature strongly suggests that a significant UCAF remains in most of the copolymers despite of the relatively high crystallinity. Interestingly, the segmental dynamics is found to be very sensitive to the details of the crystallization process, indicative of subtle changes induced by crystallization in the remaining amorphous phase.

\section{EXPERIMENTAL SECTION}

\section{Materials}

The synthesis of the PBSAz copolymers was achieved by a two-stage melt polycondensation reaction: an esterification at atmospheric pressure of succinic acid, dimethyl azelate, and 1,4-butane diol, and polycondensation at reduced pressure. Reactions were performed in a specially designed and adapted Inox Autoclave reactor (Autoclave-

France, France). The details of the synthesis have been previously reported. ${ }^{16}$ Succinic acid $(\mathrm{SuA})$ and dimethyl azelate $(\mathrm{DMAz})$ were copolymerized at $[\mathrm{COOH}]:\left[\mathrm{COOCH}_{3}\right]=1.0: 0$, $0.8: 0.2,0.6: 0.4,0.5: 0.5,0.4: 0.6,0.2: 0.8$, and $0: 1.0$ molar ratios in the presence of $1,4-$ butane diol (BDO). Samples are denoted in an abbreviated form, e.g., BSxxBAzyy, indicating the molar ratio of each component as subscripts ( $\mathrm{xx}$ and $\mathrm{yy})$.

\section{Broadband Dielectric Spectroscopy (BDS)}

Measurements of the complex dielectric permittivity $\left(\varepsilon^{*}=\varepsilon^{\prime}-\mathrm{i} \varepsilon^{\prime \prime}\right) v s$ frequency were performed in the range $10^{-1}-10^{6} \mathrm{~Hz}$, using a Novocontrol high resolution dielectric analyzer (alpha-A analyzer). The sample preparation consisted in forming a parallel plate 
capacitor filled with the sample previously melted in a hot stage under nitrogen atmosphere. An upper electrode of $20 \mathrm{~mm}$ was placed on the previously prepared film over the gold coated disk, and a separation of $100 \mu \mathrm{m}$ between both electrodes was maintained by using a cross-shaped Teflon spacer of small area. The sample cell was set in a cryostat, and its temperature was controlled via a nitrogen gas jet stream coupled with the Novocontrol Quatro controller. Before the dielectric measurements, the sample capacitor was subjected to vacuum for a few hours, and once inside the BDS cell, it was heated above the melting temperature of the polymer to erase thermal history. Isothermal frequency scans were performed every fifth degree during cooling down to $130 \mathrm{~K}$. Sample temperature was controlled by nitrogen gas flow with temperature stability better than $\pm 0.1 \mathrm{~K}$. In addition isochronal measurements at frequencies every decade between $10-10^{6} \mathrm{~Hz}$ were carried out at a $3 \mathrm{~K} / \mathrm{min}$ heating rate.

When analyzing the data, the ratio $\varepsilon^{*} / \varepsilon_{\infty}$ has been used trying to minimize the effect of the uncertainties in sample geometry when comparing results from different samples. Note that $\varepsilon_{\infty}$, as determined from very low temperature behavior at high frequencies, is free of significant dipole reorientation contributions and therefore related mainly with the induced polarization phenomena. Consequently, no significant differences in the actual $\varepsilon_{\infty}$ values among the different samples should exist.

\section{DSC Measurements}

The non-isothermal crystallization experiments for crystallinity measurements were performed by differential scanning calorimetry (DSC), using a PerkinElmer Pyris 1 calorimeter equipped with a refrigerated cooling system Intracooler 2P, under nitrogen 
atmosphere flow and calibrated with indium. The samples were weighted and sealed in aluminum pans. Experiments were generally conducted in a temperature range between 243 $\mathrm{K}$ and $418 \mathrm{~K}$ at $10 \mathrm{~K} / \mathrm{min}$ rate.

The calorimetric glass transition temperature of the copolymers was determined from the midpoint of the reversing heat flow jump in temperature-modulated experiments during heating scans at $3 \mathrm{~K} / \mathrm{min}$ rate in a temperature range between $123 \mathrm{~K}$ and $418 \mathrm{~K}$, after been cooled to $120 \mathrm{~K}$ at different rates (indicated in the text). The oscillation temperature amplitude was $0.5 \mathrm{~K}$ and the period $60 \mathrm{~s}$. The experiments were carried out using a Q2000 set up from TA Instruments with a liquid nitrogen cooling system using $25 \mathrm{~mL} / \mathrm{min}$ helium flow.

\section{RESULTS}

\section{BDS of Homopolymers}

Figure 1 presents isochronal plots of permittivity loss versus temperature at $10^{2} \mathrm{~Hz}$ for PBS and PBAz homopolymers (denoted BS and BAz). Two peaks are observed for each sample, which correspond to different kinds of molecular motions. On one hand, the $\alpha$ relaxation process, detected at high temperatures is related with the cooperative mobility of relatively large segments of the polymer, those related with the glass transition. On the other hand, the $\beta$ relaxation, detected at lower temperatures is attributed to local molecular rearrangements occurring in the glassy state. ${ }^{24}$ It should be noted that a single $\beta$ relaxation is observed for semicrystalline PBS, which is at odds with a recently published work where

an additional slower $\beta$ relaxation component was observed. ${ }^{25}$ We confirmed that this difference is attributed to the careful drying of the sample used before our dielectric 
experiments (see Supporting Information). For both PBS and PBAz, the dielectric relaxations are related with reorientations of the dipole moments in the ester groups.

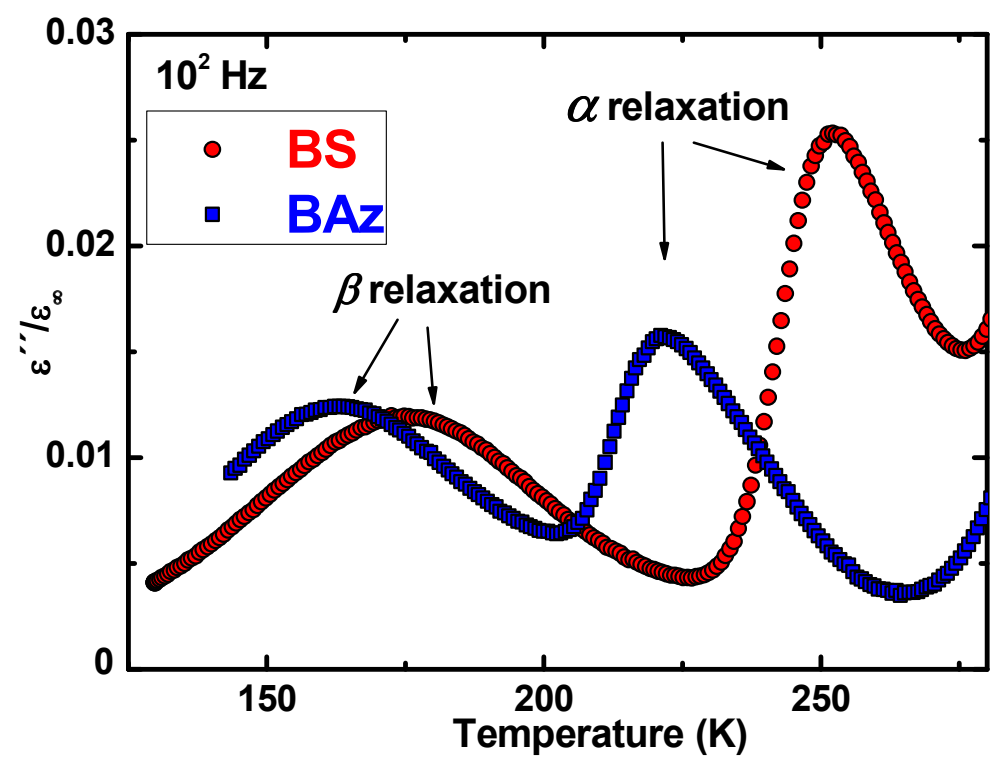

Figure 1. Isochronal plots of $\varepsilon^{\prime \prime} / \varepsilon_{\infty}$ for BS and BAz homopolymers at $10^{2} \mathrm{~Hz}$.

The isothermal permittivity loss versus frequency for the homopolymers at different temperatures, where the $\beta$ relaxation can be easily recognized, is shown in Figure $2 \mathrm{a}$. The $\mathrm{BAz} \beta$ relaxation peaks have higher intensity as compared to those corresponding to BS. Moreover, the peak frequency, $f_{\max }$, of BAz losses occurs at markedly higher frequencies than those of BS, indicating a faster local dynamics of the former. This could be attributed to the longer aliphatic part of the BAz repeating unit. In order to quantify these differences, the relaxation time from each isothermal plot has been calculated as:

$$
\tau=\left(2 \pi f_{\max }\right)^{-1}
$$


The resulting values are presented in Figure 2b. For both polymers a clear Arrhenius behavior is found and accordingly the data have been fitted to Arrhenius equation:

$$
\tau(T)=\tau_{\infty} \exp \left[\frac{E_{a}}{k_{B} T}\right]
$$

The resulting fitting parameters are shown in Table 1. The activation energy for BS, as determined from the Arrhenius fit, is $41.1 \mathrm{~kJ} / \mathrm{mol}$, which agrees very well with values previously reported, ${ }^{26,27}$ whereas the value for $\mathrm{BAz}$ is $39.2 \mathrm{~kJ} / \mathrm{mol}$. No values of activation energy for BAz have been found in literature, but the value obtained here is close to those reported for other aliphatic polyesters. ${ }^{28-32}$

As can be seen in Table 1 , the values obtained for $\tau_{\infty}$ are far smaller than those corresponding to typical vibrational frequencies, a situation that is often found when analyzing the secondary relaxations of polymers. These extremely low values are indicating that the molecular origin of the relaxation does not correspond to single activated jumps between two equivalent positions of molecular units over an energy barrier, and therefore, a more complex situation should be envisaged. One way of describing such behavior is by using the Eyring equation, where the relaxation time is expressed in terms of the difference in the Gibbs free energy, $\Delta G=\Delta H-T \Delta S$, between the equilibrium and the activated state. This leads to an equation similar to the Arrhenius one, where an entropic term appears in addition to the enthalpic one, i.e.

$$
\tau(T)=\frac{h}{k_{B} T} \exp \left[\frac{\Delta G}{k_{B} T}\right]=\frac{h}{k_{B} T} \exp \left[-\frac{\Delta S}{k_{B}}\right] \exp \left[\frac{\Delta H}{k_{B} T}\right]
$$


where $h$ is Plank's constant and $\Delta H$ and $\Delta S$ are, respectively, the enthalpic and entropic changes corresponding to the activated state. When the data in Figure $2 \mathrm{~b}$ are described in this way, nearly undistinguishable fitting, from the Arrhenius one, is found and the corresponding parameters are also shown in Table 1. As expected the entropic terms take relatively large values. When comparing the values of the entropic terms for the two polymers we found a significantly larger value for BAz, a fact indicative of more complex local arrangements. On the other hand, the enthalpic terms obtained with the Eyring analysis are about $10 \%$ smaller than the activation energies obtained from the Arrhenius fitting above but follow the same trend.

To analyze more quantitatively the $\beta$ relaxations, the main part of the loss peak has been described by a Gaussian-like function:

$$
\varepsilon^{\prime \prime}(f)=\varepsilon^{\prime \prime} \max \exp \left[\frac{\left(\log f-\log f_{\max }\right)^{2}}{w^{2}}\right]
$$

The fittings to eq. 4 are shown as solid lines in Figure 2a. In this way, the main part of the curves (data around the loss peak) are well described, although some deviations at very high and very low frequencies remains evident.

In the framework of the Eyring description used above, the full width at half maximum of this Gaussian description of $\varepsilon^{\prime \prime}(f), F W H M_{\varepsilon^{\prime \prime}}=2 \sqrt{\ln 2} w$, can be approximately related to the $F W H M$ of the corresponding distributions of the enthalpic and entropic terms in the Eyring equation as: ${ }^{33}$ 


$$
F W H M_{\varepsilon^{\prime \prime}}-1.14=\frac{\log e}{k_{B} T} F W H M_{\Delta H}-\frac{\log e}{k_{B}} F W H M_{\Delta S}
$$

The values of $F W H M_{\varepsilon^{\prime \prime}}$ obtained at different temperatures are well described by this equation and the so obtained $F W H M_{\Delta H}$ and $F W H M_{\Delta S}$ values, corresponding to the distributions of the enthalpic and entropic terms, are also included in Table 1. It is found that the distribution width of the entropic term is markedly larger for BS than for BAz, whereas those of the enthalpic terms are not so quite similar to each other.

Table 1. Parameters Describing the Temperature Dependence of the $\beta$ relaxation of the Homopolymers. Typical uncertainty values are indicated in the table head.

\begin{tabular}{|c|c|c|c|c|c|c|}
\hline$\beta$ relaxation & $\begin{aligned} & \tau_{\infty}(s) \\
\pm & 30(\%)\end{aligned}$ & $\begin{array}{c}E_{a} \pm 1 \\
(\mathrm{~kJ} / \mathrm{mol})\end{array}$ & $\begin{array}{c}\Delta H \pm 1 \\
(\mathrm{~kJ} / \mathrm{mol})\end{array}$ & $\begin{array}{c}\Delta S \pm 0.001 \\
(\mathrm{~kJ} \mathrm{~K} / \mathrm{mol})\end{array}$ & $\begin{array}{c}F W H M_{\Delta H} \\
\pm 5(\%)\end{array}$ & $\begin{array}{c}F W H M_{\Delta S} \\
\pm 5(\%)\end{array}$ \\
\hline BS & $3.4 \times 10^{-16}$ & 41 & 40 & 0.046 & 56 & 110 \\
\hline BAz & $7.3 \times 10^{-17}$ & 39 & 38 & 0.060 & 49 & 64 \\
\hline
\end{tabular}



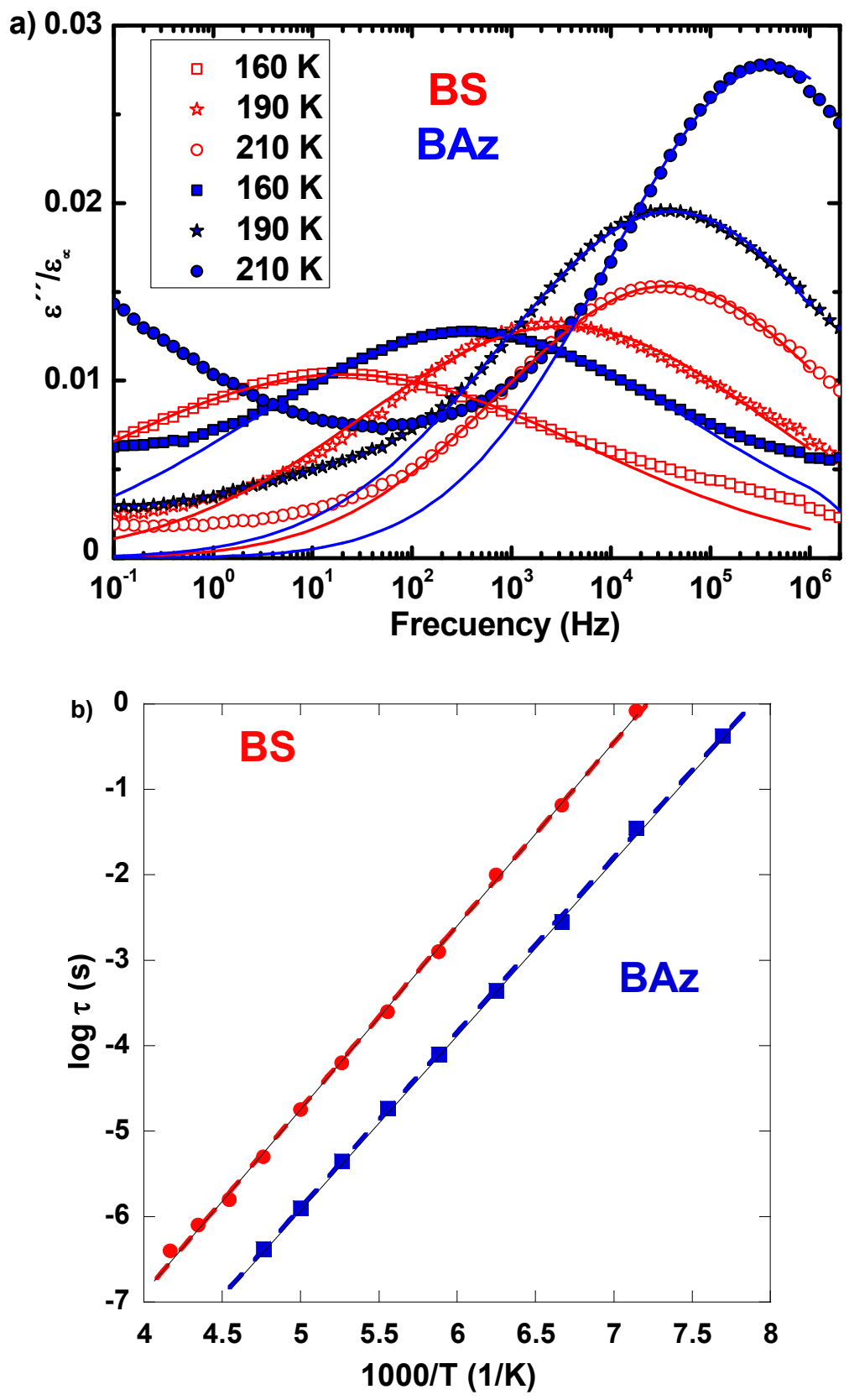

Figure 2. (a) Dielectric loss spectra versus frequency of both homopolymers at 160, 190 and $210 \mathrm{~K}$. Lines are the descriptions of the main loss peaks in terms of Gaussian like functions (b) Arrhenius plots of $\beta$ processes for BS and BAz homopolymers. Solid lines are Arrhenius fittings whereas dashed lines represent the Eyring descriptions.

Figures $3 \mathrm{a}$ and $3 \mathrm{~b}$ show representative isothermal dielectric loss curves in the temperature range relevant for the $\alpha$ relaxation. In these graphs, it can be observed that the $\alpha$ relaxation peaks have lower intensity than those for the $\beta$ relaxations at the same 
temperature and this makes difficult an accurate quantitative analysis. The relatively low values of the dielectric losses corresponding to the $\alpha$ relaxation can be attributed to the relatively high crystallinity of the polymers which simultaneously produce a broad relaxation and reduced dramatically the relaxation strength which is proportional to the area below the loss peak. ${ }^{22}$ Consequently the quantification of the $\alpha$ relaxation rate has been made by using the isochronal representation of the dielectric losses (see Figure 1 as an example). In this representation when selecting low frequencies, the $\alpha$ relaxation is observed as a clear and prominent loss peak a few degrees above $T_{\mathrm{g}}$, well separated from the secondary relaxation, which is detected at temperatures well below $T_{\mathrm{g}}$. Moreover, the usually high apparent activation energy of the $\alpha$ relaxation gives rise to a relatively sharp peak. The dramatic differences between the isothermal and isochronal representation of the $\alpha$ relaxation data is a general feature in systems where a broad distribution of relaxation times exist, as it is the case of semicrystalline polymers, polymer networks, polymer blends, etc. Using the isochronal representation of the dielectric losses at different frequencies, the temperature $\left(T_{\max }\right)$ at which the $\alpha$ relaxation loss-peak occurs has been determined for both polymers. The results obtained are plotted in Figure 3c. In this figure, the equivalent results obtained from raw data of isothermal dielectric relaxation at $250 \mathrm{~K}$ for $\mathrm{BS}$ and $220 \mathrm{~K}$ for $\mathrm{BAz}$ are included for comparison purposes (star symbols). In this way, we confirm that the differences between both types of analysis are not large.

In order to describe the temperature dependence of $\alpha$ relaxation, the data in Figure 3c were fitted to a Vogel-Fulcher-Tamman-like equation (VFT),

$$
f\left(T_{\max }\right)=f_{\infty} \exp \left(\frac{-B}{T_{\max }-T_{0}}\right)
$$


where $T_{0}$ is the Vogel temperature, $B$ is an energetic term and $f_{\infty}$ would correspond to a typical vibration frequency. The obtained fitting lines are shown in Figure $3 \mathrm{c}$ and the parameters are included in Table 2. As the $\alpha$ relaxation in BAz is rather close to the $\beta$ relaxation (see Figure 1), the loss peak temperature determined using high frequency data can be influenced significantly by contributions from the $\beta$ relaxation. Thus, in the fitting of BAz data only the low frequency range has been taken into account, and because that the value $f_{\infty}$ was taken equal to that found for BS. In this way a good description of the data of BAz is obtained below $f=10^{4} \mathrm{~Hz}$. Note that at this frequency the $\beta$ relaxation of BAz is already less than 2 decades faster than the $\alpha$ relaxation (see dotted line in Figure $3 \mathrm{c}$ ).

Here it is important to recall the connection between the $\alpha$ relaxation and the glass transition. In a semicrystalline polymer mobile dipolar entities exist only in the amorphous phase and the reorientation of the molecular dipoles is essentially blocked below the glass transition temperature. Therefore, a glass transition temperature can be determined from dielectric experiments as the temperature at which molecular mobility is extremely small. The dielectric glass transition temperature values, $T_{\mathrm{g}, \mathrm{BDS}}$, calculated using equation 6 as $f\left(T_{\mathrm{g}, \mathrm{BDS}}\right)=10^{-3} \mathrm{~Hz}$ are shown in Table 2 . As it can be seen, these values are almost the same as those determined for $T_{\mathrm{g}}$ by DSC measurements. 

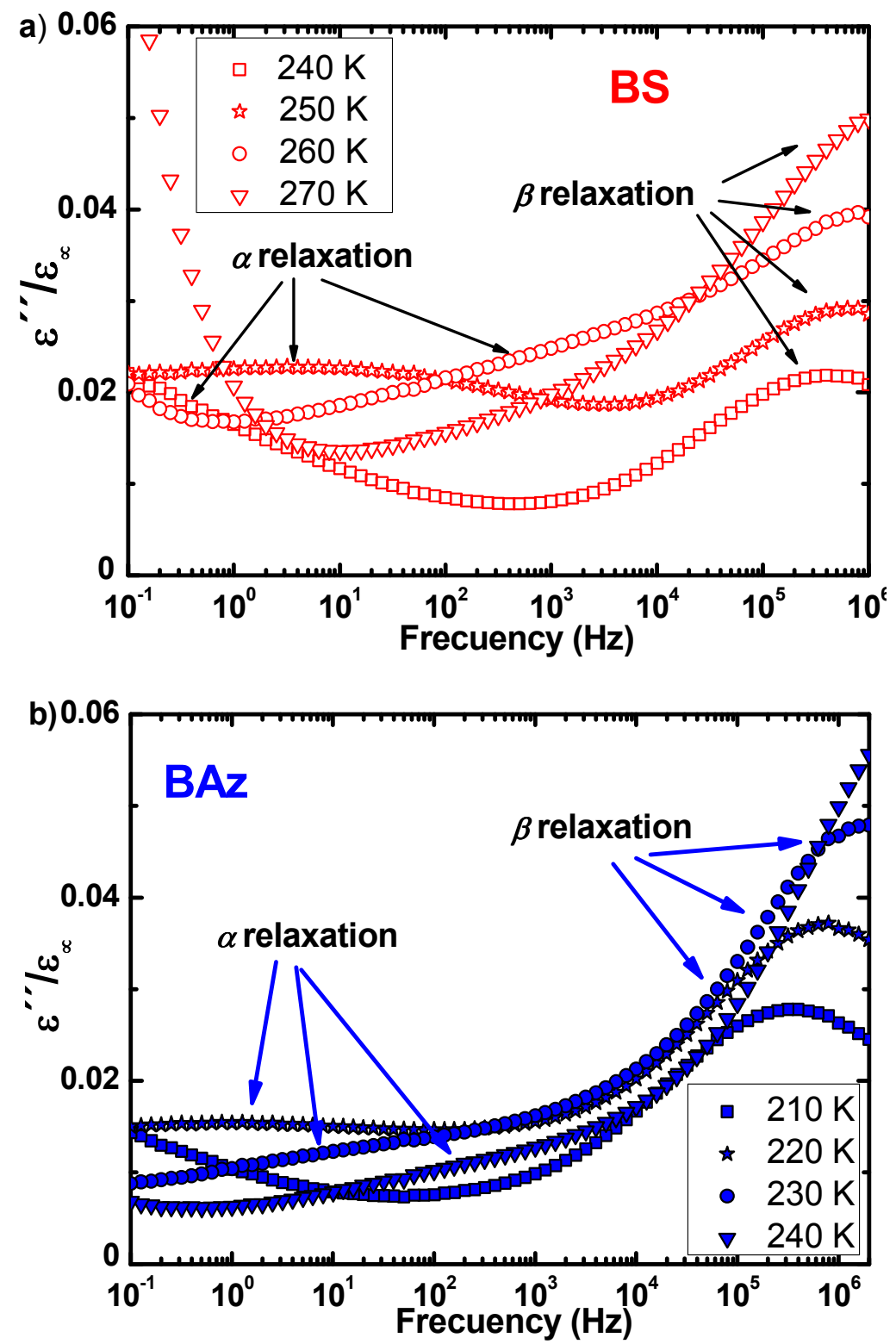


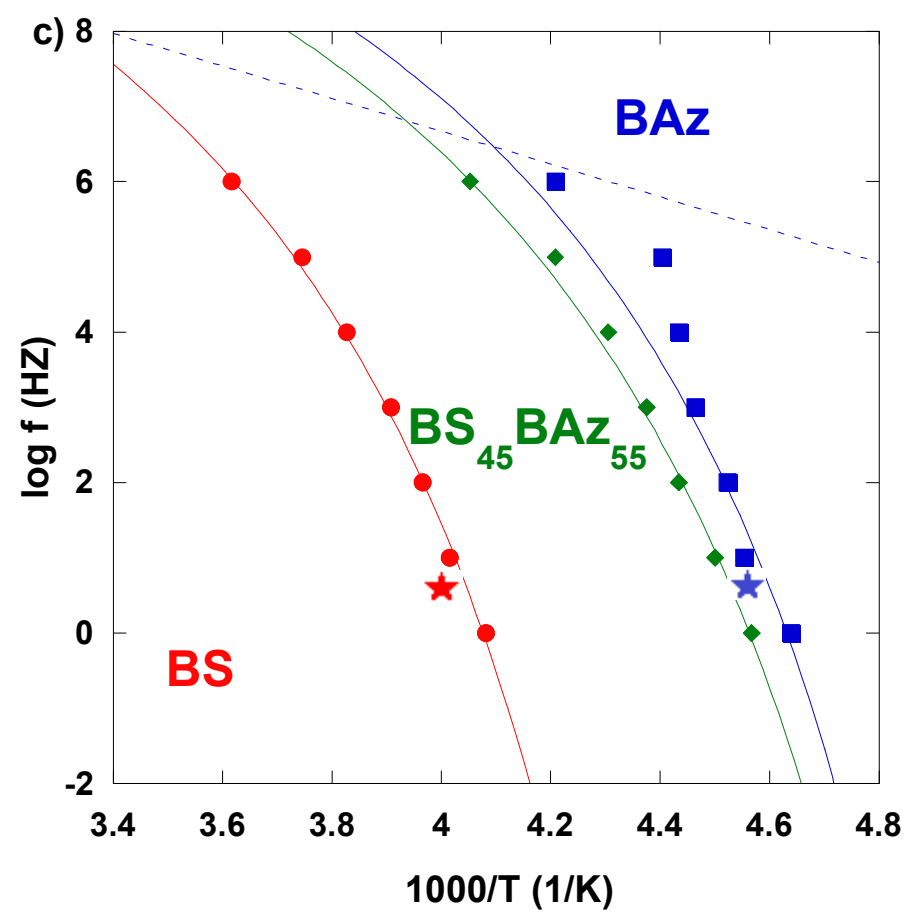

Figure 3. (a) Dielectric loss spectra versus frequency of BS homopolymer at different temperatures. (b) Dielectric loss spectra versus frequency of BAz homopolymer at different temperatures (c) Arrhenius plot of the peak frequencies of $\mathrm{BS}, \mathrm{BAz}$ and $\mathrm{BS}_{45} \mathrm{BAz} \mathrm{z}_{55}$. Solid lines represent the corresponding VFT fit of $\alpha$ relaxation, dotted line represents the Arrhenius fit of $\beta$ relaxation for the BAz homopolymer.

Table 2. Parameters Describing the Temperature Dependence of the $\alpha$ Relaxation of the Homopolymers and Glass Transition Temperatures Determined by DSC.

\begin{tabular}{ccccc|c}
\hline$\alpha$ relaxation & $\boldsymbol{B} \pm \mathbf{5}(\mathbf{K})$ & $\boldsymbol{T}_{\mathbf{0}} \pm \mathbf{1}(\mathbf{K})$ & $\boldsymbol{f}_{\infty}(\mathbf{H z})$ & $\begin{array}{c}\boldsymbol{T}_{\mathrm{g}, \mathrm{BDS}} \\
\pm \mathbf{1}(\mathbf{K})\end{array}$ & $\begin{array}{c}\boldsymbol{T}_{\mathrm{g}, \text { DSC }} \\
\mathbf{2}(\mathbf{K}) \\
\mathbf{D S C}^{(\text {(midpoint) }}\end{array}$ \\
\hline BS & 1210 & 207 & $4.5 \times 10^{13}$ & 238 & 238 \\
$\mathbf{B A z}$ & 974 & 185 & $(*) 4.5 \times 10^{13}$ & 210 & 210 \\
\hline
\end{tabular}

$(*)$ The value was taken equal to that found for BS because in the fitting of BAz data only the low frequency range was taken into account.

\section{BDS of Copolymers}

Analogous measurements have been made for all copolymer samples. Figure 4 shows the isochronal plots of $\varepsilon^{\prime \prime} / \varepsilon_{\infty}$ at relatively low frequency $\left(10^{2} \mathrm{~Hz}\right)$, where $\alpha$ and $\beta$ 
relaxation can be easily located, for all copolymer compositions. The results for both homopolymers are plotted for comparison purposes with dotted lines.

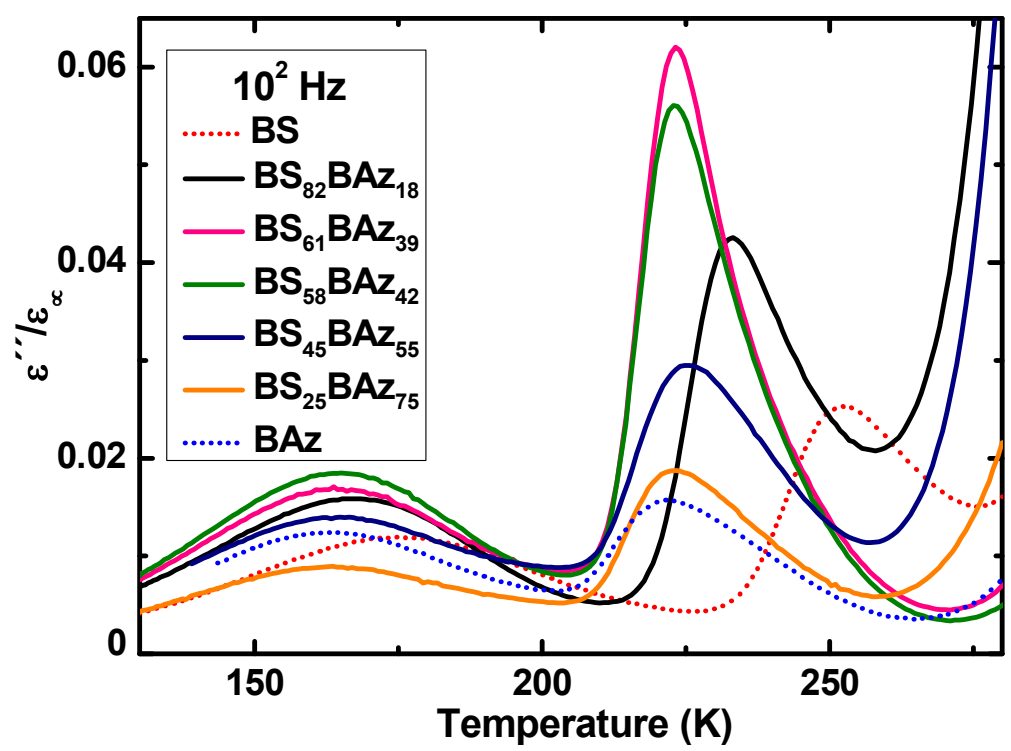

Figure 4. Isochronal plots of $\varepsilon^{\prime \prime} / \varepsilon_{\infty}$ for PBSAz copolymers at $10^{2} \mathrm{~Hz}$.

At first sight, it is obvious that the dielectric behavior of the copolymers is far from monotonous. This evidences the high sensitivity of dielectric relaxations to changes in copolymer composition, which influences simultaneously the structural characteristics (crystallinity) and the mobility of the amorphous phase.

When local dynamics are investigated in semicrystalline polymers, it is found that the dielectric relaxation strength is proportional in a good approximation to the total amorphous fraction. ${ }^{21,34}$ However, polymer crystallinity does not have a large effect (if any) neither on the characteristic time of the relaxation process nor on the shape of the loss curve. Therefore, the data in the $\beta$ relaxation range can provide quantitative information on the fraction of comonomers involved in the crystallinity of the copolymers investigated.

Figure 5 shows the isothermal $\beta$ dielectric relaxation loss spectra at $160 \mathrm{~K}$ for all materials. The dielectric results for both homopolymers are plotted with open symbols, and 
the lines correspond to the Gaussian description used above. By assuming that most local molecular motions in a copolymer remain largely unaffected with respect to those in the pure polymers, which is in agreement with the already reported results on polybutadienes with different microstructures (ranging from 1,4-polybutadyene to 1,2-polybutadyene), ${ }^{35}$ the dielectric $\beta$ relaxation in the copolymers could be expressed as:

$$
\varepsilon^{\prime \prime}{ }_{B S A z}=\left(\Phi_{m}^{B S}\right)\left(\frac{\varepsilon^{\prime \prime}{ }_{B S}}{1-X_{m B S}}\right)\left(1-R F_{B S}\right)+\left(1-\Phi_{m}^{B S}\right)\left(\frac{\varepsilon^{\prime \prime}{ }_{B A z}}{1-X_{m B A z}}\right)\left(1-R F_{B A z}\right)
$$

where $X_{m}$ corresponds to mass crystalline fraction of the homopolymer, $R F_{B S}$ and $R F_{B A z}$ accounts for the relative fraction of the corresponding comonomer incorporated in the crystalline phase, and consequently not contributing to the $\beta$ dielectric relaxation.

Once the $\beta$ relaxations of the homopolymers were properly described with the Gaussian description presented above, under this assumption, the $\beta$ relaxation of a copolymer with BS mass fraction $\Phi_{m}^{B S}$ is fully determined by the relative fractions of both comonomers $R F_{\mathrm{BS}}$ and $R F_{\mathrm{BAz}}$ incorporated in the crystalline phase. Following this method we have look for the suitable values of $R F_{\mathrm{BS}}$ and $R F_{\mathrm{BAz}}$ that provide the best description of the $\beta$ relaxation of the copolymers. The resulting values are shown in Table 3 as compared with the previously reported copolymer crystallinities obtained by DSC. ${ }^{14}$

When comparing the values obtained by DSC and BDS in Table 3, it should be noted that DSC determines the total weight fraction of segments incorporated within the crystals of the BS rich phase and/or BAz rich phase respectively, whereas BDS is sensitive to the loss of mobile comonomers irrespectively to the kind of crystals that are formed. In particular, if large co-crystallization takes place, the analysis of BDS and DSC experiments would not provide the same numbers. This is likely the case for $\mathrm{BS}_{25} \mathrm{BAz}_{75}$, where $\mathrm{BDS}$ detects a fraction of BS being involved in the crystallization but no BS like crystallization 
was detectable by DSC. ${ }^{14}$ This result is consistent with the isodimorphic nature of the copolymers, where a small fraction of the minority comonomer is incorporated in the crystalline unit cells of the majority component, as previously demonstrated by WAXS and thermal fractionation by some of us. ${ }^{14,15}$ Despite the different sensitivities of DSC and BDS to crystallization phenomena, a remarkable good agreement between the crystallized mass fraction of each comonomer and the amount of the corresponding crystalline phase is found in most of the copolymers. This result is consistent with the already reported fact that the amount of comonomer exclusion is in general much larger than the amount of comonomer inclusion during crystallization of these copolymers. ${ }^{14,15}$

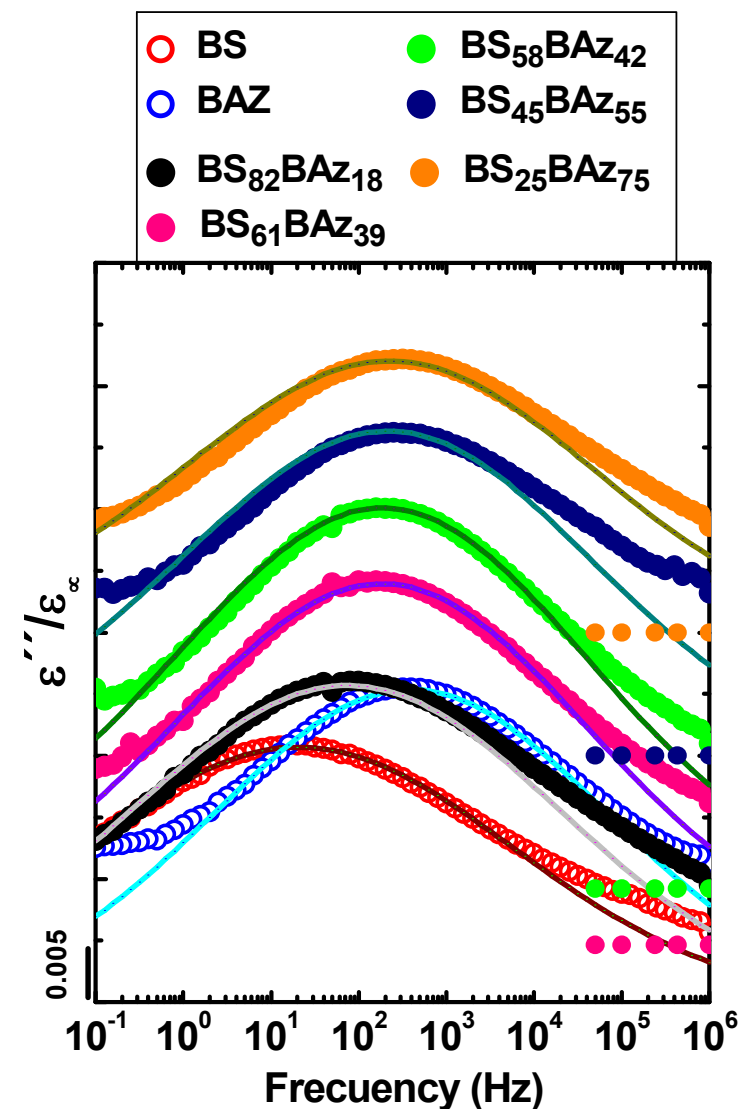

Figure 5. Calculations of new dielectric loss spectra versus frequency at $160 \mathrm{~K}$. Homopolymers and $\mathrm{BS}_{82} \mathrm{BAz}_{18}$ are without vertical displacement. For the sake of clarity, losses of the other compositions are vertically shifted and the corresponding zero level displayed as a dotted line. 
Table 3. Comonomer Relative Mass Fractions in the Crystalline Phase $( \pm 0.01)$ Deduced from BDS and Mass Fractions in the Different Crystalline Forms $( \pm 0.01)$ Determined by DSC. $^{14}$

\begin{tabular}{ccccccc}
\hline & $\Phi_{\boldsymbol{m o l}}^{\boldsymbol{B S}}$ & $\Phi_{\boldsymbol{m}}^{\boldsymbol{B S}}$ & $\boldsymbol{R F}_{\mathbf{B S}}$ & $\boldsymbol{R} \boldsymbol{F}_{\mathbf{B A z}}$ & $\boldsymbol{X}_{\mathbf{m P B S}}$ & $\boldsymbol{X}_{\mathbf{m P B A z}}$ \\
\hline $\mathbf{B S}$ & 1 & 1 & 0.32 & - & 0.32 & - \\
$\mathbf{B S}_{\mathbf{8 2}} \mathbf{B A z}_{\mathbf{1 8}}$ & 0.82 & 0.76 & 0.32 & 0 & 0.32 & 0 \\
$\mathbf{B S}_{\mathbf{6 1}} \mathbf{B A z}_{\mathbf{3 9}}$ & 0.61 & 0.53 & 0.43 & 0 & 0.40 & 0 \\
$\mathbf{B S}_{\mathbf{5 8}} \mathbf{B A z}_{\mathbf{4 2}}$ & 0.58 & 0.50 & 0.38 & 0 & 0.38 & 0 \\
$\mathbf{B S}_{\mathbf{4 5}} \mathbf{B A z}$ & 0.45 & 0.37 & 0.40 & 0.28 & 0.38 & 0.27 \\
$\mathbf{B S}_{\mathbf{2 5}} \mathbf{B A z}$ & 0.25 & 0.19 & 0.1 & 0.52 & 0 & 0.54 \\
$\mathbf{B A z}$ & 0 & 0 & - & 0.33 & - & 0.33 \\
\hline
\end{tabular}

Concerning the $\alpha$ relaxation, the situation is more complex, because as aforementioned all the characteristics of the $\alpha$ relaxation are strongly affected by crystallinity, the relaxation strength is dramatically reduced, the relaxation time becomes much longer and the relaxation curves extend over large frequency ranges. Moreover, in crystallizable copolymers the fraction of comonomer involved in the different amorphous fractions RAF, CAF and UCAF cannot be anticipated. As can be seen in Figure 4, when increasing the BS fraction in the copolymers with respect to BAz homopolymer, there is a clear increase in the dielectric loss intensity, without much change in the peak position. The former would be related with the decreasing crystallinity of the copolymers, whereas the later possibly indicates that the more mobile amorphous fraction of the copolymers is rich in BAz comonomers. This trend continues up to the copolymer with the highest BS fraction (82\% molar fraction), where a clear drop in intensity and a peak shift is detected.

The behavior described in the previous paragraph is in qualitative agreement with the output of the analysis of the $\beta$ relaxation summarized in Table 3 . For example, see Table 4, for $\mathrm{BS}_{82} \mathrm{BAz}_{18}$ the $\mathrm{BS}$ mass fraction within the copolymer in amorphous fractions $\varphi_{A}^{B S}$ is 0.51 and that of $\mathrm{BAz}\left(\varphi_{A}^{B A z}\right)$ is 0.24 . Conversely, for $\mathrm{BS}_{61} \mathrm{BAz}_{39}$ the $\mathrm{BS}$ mass fraction 
in the copolymer in the amorphous fractions $\left(\varphi_{A}^{B S}\right)$ is 0.30 and that of $\mathrm{BAz}\left(\varphi_{A}^{B A z}\right)$ is 0.47 . These differences can explain the quite dramatic changes in the $\alpha$ relaxation between both copolymers. Moreover, as described above the 3 different amorphous fractions (RAF, CAF and UCAF) that can exist in each copolymer sample could present different fractions of the two comonomers, with no clear reasons a priori for any trend.

Table 4. Total ( $\left.\Phi_{\mathrm{m}}\right)$ and Amorphous $\left(\varphi_{\mathrm{A}}\right)$ Mass Fractions of BS and BAz within the

\begin{tabular}{cccc}
\multicolumn{4}{c}{ Copolymers $( \pm 0.01)}$. \\
\hline & $\Phi_{\boldsymbol{m}}^{\boldsymbol{B S} / \mathbf{B A z}}$ & $\boldsymbol{\varphi}_{\boldsymbol{A}}^{\boldsymbol{B S}}$ & $\boldsymbol{\varphi}_{\boldsymbol{A}}^{\boldsymbol{B A z}}$ \\
\hline $\mathbf{B S}$ & $0 / 1$ & 0.68 & - \\
$\mathbf{B S}_{\mathbf{8 2}} \mathbf{B A z}_{18}$ & $0.76 / 0.24$ & 0.51 & 0.24 \\
$\mathbf{B S}_{\mathbf{6 1}} \mathbf{B A z}_{\mathbf{3 9}}$ & $0.53 / 0.47$ & 0.30 & 0.47 \\
$\mathbf{B S}_{\mathbf{5 8}} \mathbf{B A z}_{42}$ & $0.50 / 0.50$ & 0.31 & 0.50 \\
$\mathbf{B S}_{\mathbf{4 5}} \mathbf{B} \mathbf{A} z_{55}$ & $0.37 / 0.63$ & 0.22 & 0.45 \\
$\mathbf{B S}_{\mathbf{2 5}} \mathbf{B A z}$ & $0.19 / 0.81$ & 0.17 & 0.39 \\
$\mathbf{B A z}$ & $0 / 1$ & - & 0.67 \\
\hline
\end{tabular}

Due to the difficulties mentioned above for the homopolymers, the quantitative analysis of the $\alpha$ relaxation time in the copolymers has also been made by using the isochronal data representations (as those shown in Figure 4). In this way, the glass transition temperature determined from BDS has been calculated for the copolymers following the same procedure used for the homopolymers, i.e., calculating by means of the VFT fit the temperature at which the peak loss would occur for a frequency of $10^{-3} \mathrm{~Hz}$, see copolymer data in Figure $3 \mathrm{c}$ as an example. As for BAz, the value of $f_{\infty}$ was fixed and only the low frequency data $\left(f<10^{4} \mathrm{~Hz}\right)$ were used in the fitting. The $T_{g \text {,BDS }}$ results are shown in Figure 6 as a function of BAz fraction in comparison with glass transition temperatures determined by calorimetry $T_{\mathrm{g}, \mathrm{DSC}}$. 
When selecting the horizontal axes of Figure 6 for a mixture of partially crystalline polymers, several circumstances have to be taken into account. On one hand, the crystalline fraction does not participate directly in the glass transition phenomena, although it can have a significant impact in the glass transition temperature of the surrounding amorphous phase.

On the other hand, the amorphous regions in semicrystalline polymers tend to have some polymer strands with short range local order packing that apparently does not contribute to the glass transition as detected by DSC, ${ }^{36}$ but can also affect the value of the glass transition temperature. Our choice in Figure 6 has been using as horizontal axis the mass fraction of amorphous BAz with respect to the total amorphous phase in the copolymer. These values have been determined from the output of the analysis of the dielectric $\beta$ relaxation results shown above.

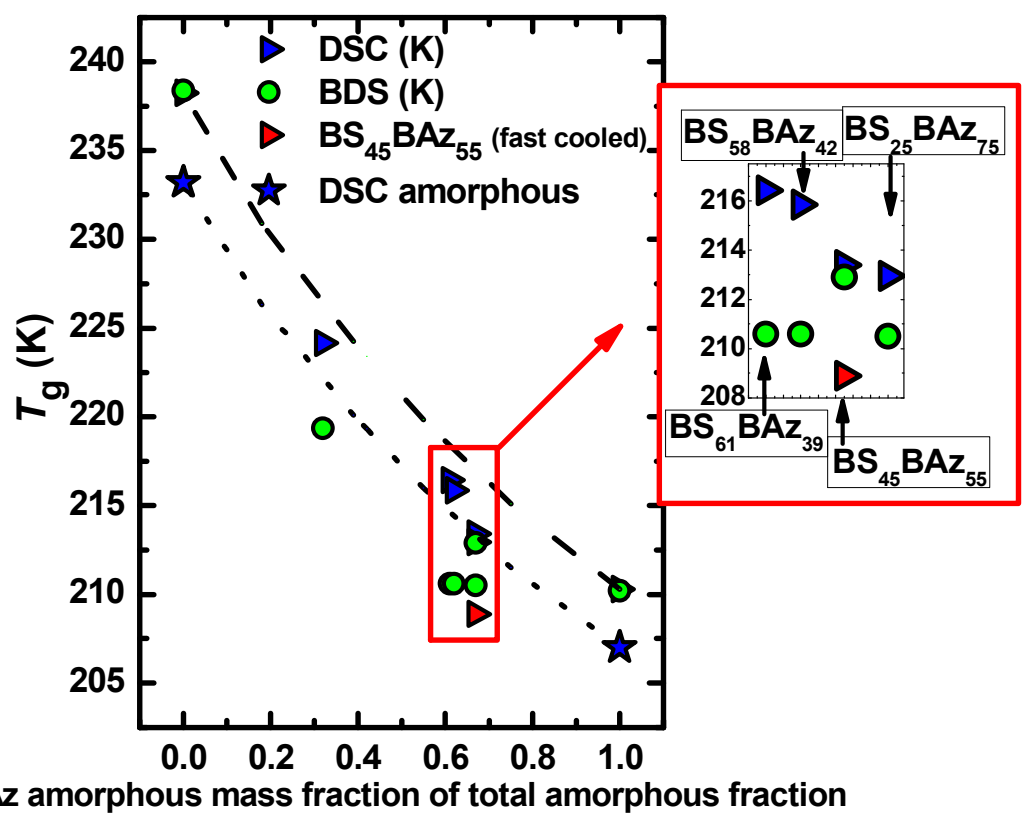

Figure 6. Glass transition temperatures as a function of weight fraction of amorphous BAz with respect to the total amorphous phase. Dashed line and dotted lines correspond to the Fox equation with the glass transition of semicrystalline and amorphous polymers respectively. 
The values of $T_{\mathrm{g}, \mathrm{DSC}}$ lie close to the prediction of the Fox equation obtained from the values of the $T_{\mathrm{g}, \mathrm{DSC}}$ of the homopolymers (see dashed line in Figure 6). However, significant differences between $T_{\mathrm{g}, \mathrm{BDS}}$ and $T_{\mathrm{g}, \mathrm{DSC}}$ are found in most of the copolymers. $T_{\mathrm{g}, \mathrm{BDS}}$ values are generally $5 \mathrm{~K}$ lower than those determined by DSC. Nevertheless, for $\mathrm{BS}_{45} \mathrm{BAz}_{55}$ (whose amorphous BAz mass fraction with respect to the total amorphous phase is 0.67 ) a rather good agreement between $T_{\mathrm{g}, \mathrm{BDS}}$ and $T_{\mathrm{g}, \mathrm{DSC}}$ is found, as it was also the case for the homopolymers.

As already commented, differences between $T_{\mathrm{g}, \mathrm{BDS}}$ and $T_{\mathrm{g}, \mathrm{DSC}}$ can be explained by taking into account the effects of the crystalline regions on the mobility of the remaining amorphous phase. In a recent work on poly(butylene succinate), ${ }^{25}$ it has been found that the glass transition temperature of a fully amorphous PBS (blue star symbol in Figure 6) is about $6 \mathrm{~K}$ smaller than that of crystallized PBS. As far as we are aware, no corresponding value for PBAz has been reported. Thus, in order to obtain a $T_{\mathrm{g}, \mathrm{DSC}}$ value for amorphous BAz (yellow star symbol in Figure 6), a new DSC experiment was performed on a sample of the homopolymer previously cooled at a high rate by quenching the polymer melt in liquid nitrogen. The subsequent heating scan exhibits a clear step in heat flow immediately followed by cold-crystallization peak corroborating that a measurable amount of unconstrained amorphous fraction (UCAF) was obtained in the sample during the fast cooling. The so obtained $T_{\mathrm{g}, \mathrm{DSC}}$ value is about $3.5 \mathrm{~K}$ smaller than that of crystallized PBAz. In line with these results, dielectric experiments on poly(propylene succinate) (PPS) ${ }^{30}$ (a polymer belonging to the same family as PBAz and PBS) provided an estimate of the difference in glass transition temperature of about $5 \mathrm{~K}$ when comparing data of fully amorphous PPS and those of PPS with $20 \%$ crystallinity. It is noteworthy that these differences are about the same as those observed between $T_{\mathrm{g}, \mathrm{BDS}}$ and $T_{\mathrm{g}, \mathrm{DSC}}$ results in most 
of the copolymers, particularly when BAz is not involved in crystallization, i.e., in the copolymers $\mathrm{BS}_{82} \mathrm{BAz}_{18}, \mathrm{BS}_{61} \mathrm{BAz}_{39}$, and $\mathrm{BS}_{58} \mathrm{BAz}_{42}$.

As the BDS values of $T_{\mathrm{g}}$ have been determined from the peak position of the dielectric losses, these values will most probably reflect the segmental mobility in the most mobile amorphous fraction. If only two amorphous fractions would exist in a polymer material (RAF and CAF) the $T_{g}$ value would reflect the mobility of the CAF, however, in a situation where also an unconstrained amorphous fraction (UCAF) exists, ${ }^{23}$ the dominant dielectric signal would correspond to the later. Taking this into account, we have evaluated the prediction of the glass transition temperatures for fully amorphous mixtures of BS and BAz using the Fox equation with the values of $T_{\mathrm{g}}$ for amorphous PBS and PBAz given above $(232 \mathrm{~K}$ and $207 \mathrm{~K}$ respectively). The resulting values for the copolymers are presented in Figure 6 as a dotted line. Interestingly, the $T_{\mathrm{g}, \mathrm{BDS}}$ values of the copolymers lie in general below but close the Fox prediction for fully amorphous copolymers (see Figure 6), suggesting that a possible explanation of the differences found between BDS and DSC $T_{\mathrm{g}}$ values would be that in the copolymers where BAz is not involved in the crystallization, a significant amount of UCAF is present, where no relevant effects of the crystalline phase on the molecular motions exist. This phase would present a prominent dielectric relaxation because of the absence of any constrain in the dipole moment reorientation and therefore it would determine the temperature of the loss peak. The stretched high temperature tail in the dielectric loss curves would be due to the CAF contributions. On the contrary, the DSC values are determined from the midpoint of the heat flow jump and they would be sensitive to the molecular motions occurring in the majority CAF, which are slower because of the influence of crystallinity, and the corresponding $T_{\mathrm{g}}$ values would be shifted to higher temperatures. 
A similar difference between $T_{\mathrm{g}}$ values is also present for the copolymer with higher BAz content $\left(\mathrm{BS}_{25} \mathrm{BAz}_{75}\right)$, a sample containing about $50 \%$ of $\mathrm{BAz}$ chains in the crystalline phase. In this sample, the low values of the dielectric $T_{\mathrm{g}}$ would be also indicative of the fact that a significant amount of UCAF is present. Again, the fact that the $T_{\mathrm{g}}$ value from DSC occurs at higher temperature would be explained by the slower motions in the CAF that are not influencing much the position of the dielectric loss peak.

$\mathrm{BS}_{45} \mathrm{BAz}_{55}$ is the only copolymer where $T_{\mathrm{g}, \mathrm{BDS}}$ and $T_{\mathrm{g}, \mathrm{DSC}}$ nearly coincide and this would mean that no significant UCAF exists in this copolymer, which is congruent with the presence in this copolymer of two distinct crystalline phases each rich in one of the comonomers. ${ }^{14}$ In a previous work ${ }^{14}$ it was found that in $\mathrm{BS}_{45} \mathrm{BAz}_{55}$ copolymer non-trivial differences in the crystalline state appear, depending on the thermal history used during crystallization. When the melted copolymer was cooled-down at relatively high rates, both BS-rich phase and BAz-rich phase crystallization occurs. However, by self-nucleation of the BS-rich phase the crystallization of the BAz-rich phase was strongly reduced due to the fact that BAz crystallization had to occur in the interlamellar domains of BS-rich phase spherulites, (Figure 14 in reference 14). Trying to determine the influence of the crystallization process on the dielectric relaxation results, new BDS experiments were performed on a $\mathrm{BS}_{45} \mathrm{BAz}_{55}$ copolymer crystallized using different protocols, namely different cooling rates $(10$ and $50 \mathrm{~K} / \mathrm{min})$ and also promoting BS-rich phase self-nucleation (annealing at $333 \mathrm{~K}$ during $5 \mathrm{~min}$ ). Representative curves obtained after different treatments for $\mathrm{BS}_{45} \mathrm{BAz}_{55}$ are shown in Figure $7 \mathrm{a}$. When comparing the different curves, the strong impact of the way the sample crystallizes on the dielectric relaxation is evident. At low temperatures, the isochronal plots on fast cooled samples display a weaker $\beta$ 
relaxation, which implies a higher crystallinity. The quantification of the crystalline fraction of the different comonomers in these samples, see Table 5, was obtained following the same procedure described above. In this way, we obtained high values of the BAz fraction in the crystalline phase $\left(R F_{B S}\right.$ up to 0.39$)$ for the fast cooled sample with only slightly smaller BS fraction in the crystalline phase $\left(R F_{B A z} 0.37\right.$ vs 0.40$)$. Similarly, the analysis of the melting process by DSC experiments on samples subjected to similar thermal histories, shown in Figure $7 \mathrm{~b}$, also evidenced an increasing BAz crystallinity when the cooling rate is increased.

The previous results show that fast cooling promotes BAz-rich phase crystallization most likely because of the limited development of BS crystals. This is in agreement with the interpretation of the aforementioned DSC experiments involving BS-rich phase selfnucleation, where the opposite situation (good development of the BS crystallinity) takes place and the crystalline relative fraction of BAz decreases from 0.32 to 0.25 (Table 5). Thus, when the PBS-rich phase is self-nucleated, it crystallizes to saturation at higher temperatures before the PBAz-rich phase starts to crystallize, and therefore the PBAz-rich crystallization is mainly restricted to the interlamellar domains of PBS-rich phase spherulites which are fully grown and impinged with one another. When considering the corresponding effects on the $\alpha$ relaxation, rather dramatic changes among the experimental data recorded on the same copolymer composition (i.e., $\mathrm{BS}_{45} \mathrm{BAz}_{55}$ ) were found after using different cooling rates. Namely, for the samples cooled fast enough (at 10 and $50 \mathrm{~K} / \mathrm{min}$ ) the peak intensity (Figure 7a) markedly increases and the peak occurs at significantly lower temperatures. These characteristics suggest that in these crystallization conditions a significant amount of UCAF exists. The new peak position in this copolymer agrees well 
with that determined for those copolymers with lower $\mathrm{BAz}$ amount $\left(\mathrm{BS}_{58} \mathrm{BAz}_{42}\right.$ and $\mathrm{BS}_{61} \mathrm{BAz}_{39}$ ) but showing no evidence of $\mathrm{BAz}$ comonomer crystallization (see Figure 6 and the insert showing a close up of the region around the $\mathrm{BS}_{45} \mathrm{BAz}_{55}$ copolymer data). In agreement with this result, also the glass transition temperature as determined by these new BDS experiments in fast cooled $\mathrm{BS}_{45} \mathrm{BAz}_{55}$ copolymer samples lies about $5 \mathrm{~K}$ below that determined by $\mathrm{BDS}$ in the originally measured $\mathrm{BS}_{45} \mathrm{BAz}_{55}$ copolymer (See Figure 6). In spite of that, the value of $T_{\mathrm{g}, \mathrm{DSC}}$ in the fast cooled $\mathrm{BS}_{45} \mathrm{BAz}_{55}$ copolymer remains approximately the same, as shown in Figure 7c. All this is in line with the idea that in addition to RAF and CAF a significant UCAF would be present in $\mathrm{BS}_{45} \mathrm{BAz}_{55}$ copolymer when it is cooled rapidly from the melt, whereas, if present, it would not be relevant when slow BS crystallization is allowed to develop before BAz crystallization can take place. This would be the situation of the sample subjected to self-nucleation procedure and also that of the copolymer cooled down slowly (at a rate smaller than ca. $5 \mathrm{~K} / \mathrm{min}$ ).

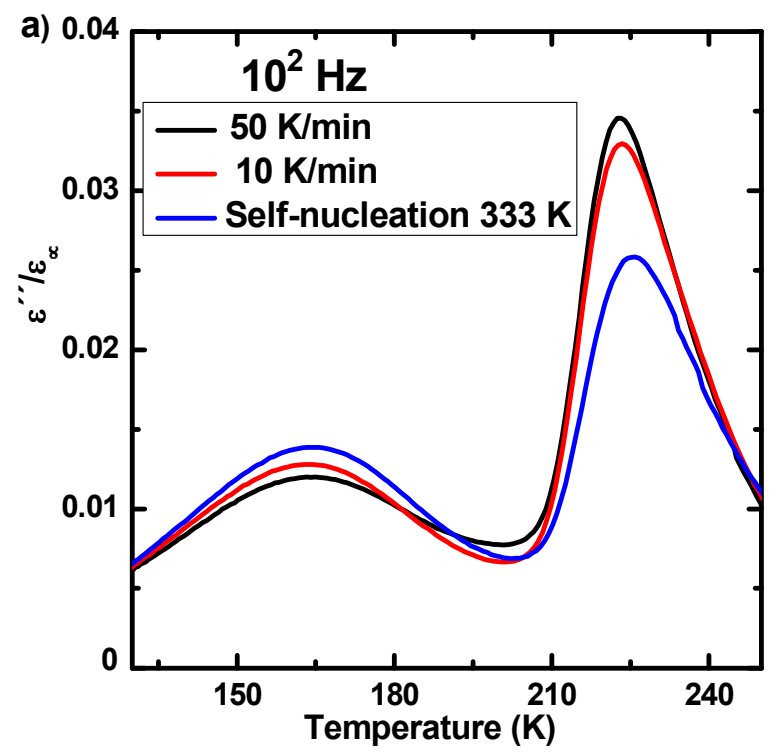



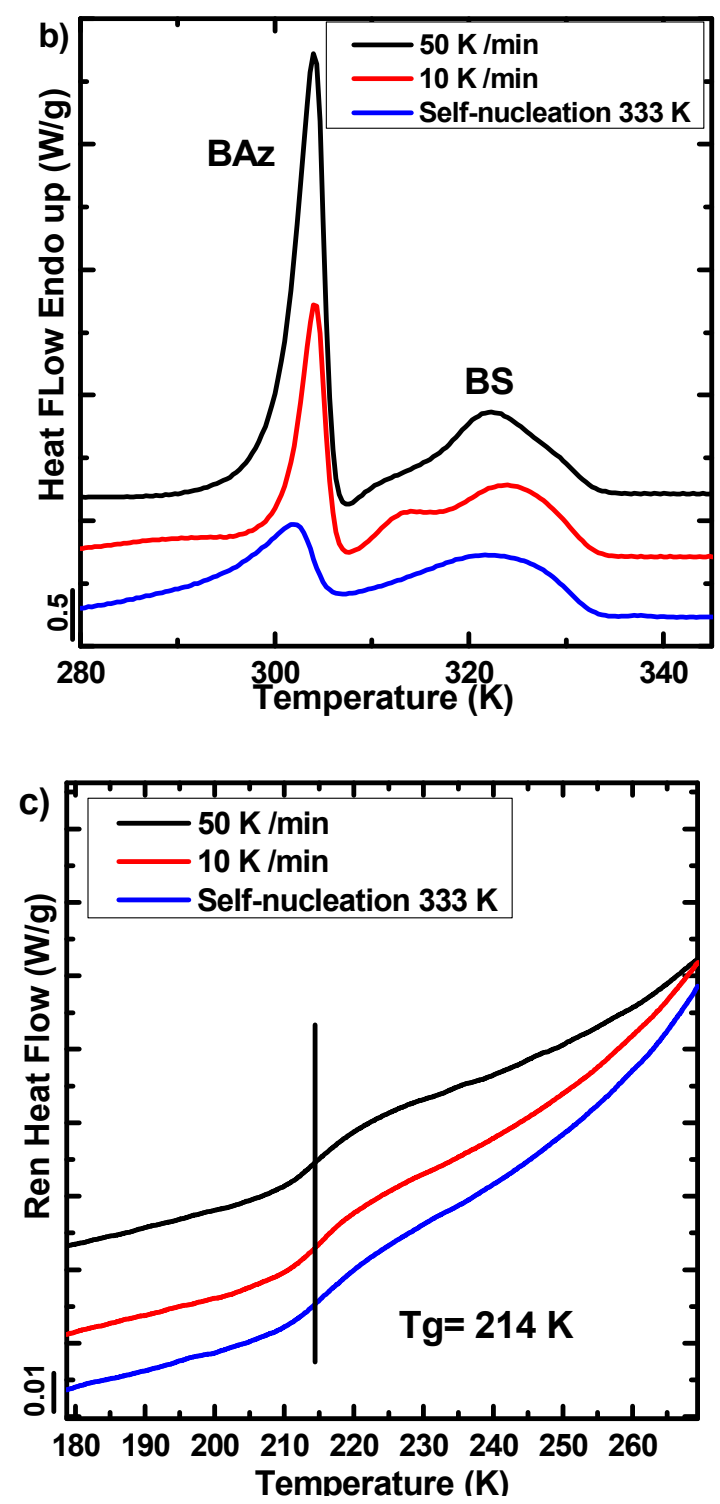

Figure 7. (a) Isochronal plots of $\varepsilon^{\prime \prime} / \varepsilon_{\infty}$ for $\mathrm{BS}_{45} \mathrm{BAz}_{55}$ copolymer at different cooling protocols $\left(10^{2} \mathrm{~Hz}\right)$ for double crystalline $\mathrm{BS}_{45} \mathrm{BAz}_{55}$ after being cooled at different rates. And corresponding DSC scans: (b) using a heating rate of $10 \mathrm{~K} / \mathrm{min}$ that exhibits both BSrich phase and BAz-rich phases melting peaks, (c) using a heating rate of $3 \mathrm{~K} / \mathrm{min}$ which shows the $T_{\mathrm{g}, \mathrm{DSC}}$ curves.

All previous results concerning the glass transition temperature as determined by BDS and DSC manifest the structural complexity of double crystalline random copolymers. In the particular case investigated here each crystalline phase is rich in one of the comonomers as was evidenced by previous studies. In addition of the crystalline phases, three distinct amorphous fractions (RAF, CAF and UCAF) exist in most copolymers. Two 
of them, i.e., CAF and UCAF, contribute to the calorimetric $T g$, while UCAF dominates the dielectric relaxation loss peak position defining the dielectric $T$ g. Finally, all three amorphous fractions (RAF, CAF and UCAF) contribute to the local dielectric secondary relaxation.

Table 5. $\mathrm{BS}_{45} \mathrm{BAz}_{55}$ Mass Fractions in the Crystalline Phase Deduced from BDS $( \pm 0.01)$ and Mass Fractions in the Different Crystalline Forms Determined by DSC $( \pm 0.01)$, Using Different Crystallization Protocols.

\begin{tabular}{|c|c|c|c|c|}
\hline $\begin{array}{c}\text { Crystallization } \\
\text { Protocol }\end{array}$ & $R F_{B S \text { (BDS) }}$ & $R F_{B A z}$ (BDS) & $X_{\text {mPBS (DSC) }}$ & $X_{\mathrm{mPBAz}(\mathrm{DSC})}$ \\
\hline $50 \mathrm{~K} / \mathrm{min}$ & 0.37 & 0.39 & 0.36 & 0.43 \\
\hline $10 \mathrm{~K} / \mathrm{min}$ & 0.37 & 0.32 & 0.36 & 0.33 \\
\hline $\begin{array}{c}\text { Self-nucleation } \\
333 \mathrm{~K}\end{array}$ & 0.40 & 0.25 & 0.33 & 0.30 \\
\hline
\end{tabular}

As can be seen in Figure 6 the data points obtained by DSC when represented as a function of the amorphous BAz content with respect to the total amorphous content of the copolymer lie below the Fox equation predictions obtained using the glass transition temperatures of the semicrystalline homopolymers. A possible explanation for the deviation would be that the RAF is relatively enriched in BS comonomers and consequently the mobile amorphous fractions would be enriched in BAz comonomers. When considering the $T \mathrm{~g}$,BDs values, the comparison with the Fox equation predictions using the glass transition temperatures of the amorphous homopolymers also show a similar trend, which again suggests that the UCAF would be enriched in BAz comonomer. Moreover, the results obtained in the $\mathrm{BS}_{45} \mathrm{BAz}_{55}$ copolymer crystallized using different protocols indicate that the amount of the phases/fractions and the comonomer partitioning in each of them can be very sensitive to the sample crystallization details. 


\section{CONCLUSIONS}

Dielectric relaxation of PBS-ran-PBAz random copolymers provides detailed information on the dynamics of the comonomers remaining in the amorphous phase. The characteristics of the more local molecular motions determine the dielectric $\beta$ relaxation for both homopolymers and copolymers, which is in agreement with previously reported results on polybutadienes with different microstructures. ${ }^{35}$ This result allows using the relaxation strength of the dielectric $\beta$ relaxation to quantify the comonomer fraction involved in the crystalline and amorphous phases, with results in overall good agreement with crystallinity values determined from DSC experiments. This agreement confirms that in these copolymers the amount of comonomer exclusion is much larger than the amount comonomer inclusion during crystallization.

On the other hand, we found that the $\alpha$ relaxation of the copolymers depends not only on the crystalline fraction but also on the details of the crystallization process. In most cases, the peak temperature of the $\alpha$ relaxation determined at low frequencies reflects in general segmental motions that are not much affected by the constrains imposed by the neighboring crystals. The relatively low values of the resulting dielectric glass transition temperatures, when compared with the calorimetric glass transition temperatures, suggest

the presence of three distinct amorphous fractions in most of the copolymers. Particularly, there is a significant amount of unconstrained amorphous fraction (UCAF) dominating the dielectric loss peak position. Nevertheless, it seems that there is a majority constrain amorphous fraction (CAF) determining the calorimetric glass transition. Experiments also showed that the multiphase character and the corresponding comonomer fractions can be influenced by the details of the crystallization protocol used. To conclude, it is noteworthy 
of remark that only by the combination of experimental techniques sensitive to different aspects of the complex structure of random copolymers formed with crystallizable comonomers it is possible to access the details of the structural organization of these extremely complex polymeric materials.

\section{ACKNOWLEDGEMENTS}

I. A. acknowledges the fellowship from the University of the Basque Country UPV/EHU. Financial support is acknowledged from UPV/EHU (UFI11/56), "UPV/EHU Infrastructure: INF 14/38"; "Mineco/FEDER: SINF 130I001726XV1/Ref: UNPV13-4E-1726" and “Mineco MAT2014-53437-C2-P”. R. M. gratefully acknowledges financial support from the Wallonia and European Commission in the frame of the BIOMAT/FEDER project, and the Interuniversity Attraction Pole program of the Belgian Federal Science Policy Office (PAI 7/05). A.A. acknowledges financial support from the Projects MAT2015-63704-P (Spanish-MINECO and EU) and IT-654-13 (Basque Government).

\section{REFERENCES}

(1) Shah, A. A.; Hasan, F.; Hameed, A.; Ahmed, S. Biological degradation of plastics: A comprehensive review. Biotechnol. Adv. 2008, 26, 246-265.

(2) Mochizuki, M.; Hirami, M. Structural Effects on the Biodegradation of Aliphatic Polyesters. Polym. Adv. Technol. 1997, 8, 203-209.

(3) Ikada, Y.; Tsuji, H. Biodegradable polyesters for medical and ecological applications. Macromol. Rapid Commun. 2000, 21, 117-132.

(4) Díaz, A.; Katsarava, R.; Puiggali, J. Synthesis, Properties and Applications of Biodegradable Polymers Derived from Diols and Dicarboxylic Acids: From Polyesters to Poly(ester amide)s. Int. J. Mol. Sci. 2014, 15, 7064-7123. 
(5) Fujimaki, T. Processability and properties of aliphatic polyesters, 'BIONOLLE', synthesized by polycondensation reaction. Polym. Degrad. Stab. 1998, 59, 209-214.

(6) Yokohara, T.; Yamaguchi, M. Structure and properties for biomass-based polyester blends of PLA and PBS. Eur. Polym. J. 2008, 44, 677-685.

(7) Chen, G. Q.; Patel, M. K. Plastics Derived from Biological Sources: Present and Future: A Technical and Environmental Review. Chem. Rev. 2012, 112, 2082-2099.

(8) Xu, J.; Guo, B. H. Microbial Succinic Acid, Its Polymer Poly(butylene succinate), and Applications. In Plastics from Bacteria: Natural Functions and Applications, Chen, G. Q., Ed.; Springer: Berlin, Heidelberg, 2010; Vol. 14, p 347.

(9) Ren, M.; Song, J.; Song, C.; Zhang, H.; Sun, X.; Chen, Q.; Zhang, H.; Mo, Z. Crystallization kinetics and morphology of poly(butylene succinate-co-adipate). J. Polym. Sci., Part B: Polym. Phys. 2005, 43, 3231-3241.

(10) Mochizuki, M.; Mukai, K.; Yamada, K.; Ichise, N.; Murase, S.; Iwaya, Y. Structural effects upon enzymatic hydrolysis of poly(butylene succinate-co-ethylene succinate)s. Macromolecules 1997, 30, 7403-7407.

(11) Zeng, J. B.; Huang, C. L.; Jiao, L.; Lu, X.; Wang, Y. Z.; Wang, X. L. Synthesis and Properties of Biodegradable Poly(butylene succinate-co-diethylene glycol succinate) Copolymers. Ind. Eng. Chem. Res. 2012, 51, 12258-12265.

(12) Diaz, A.; Franco, L.; Estrany, F.; del Valle, L. J.; Puiggali, J. Poly(butylene azelate-cobutylene succinate) copolymers: Crystalline morphologies and degradation. Polym. Degrad. Stab. 2014, 99, 80-91.

(13) Diaz, A.; Franco, L.; Puiggali, J. Study on the crystallization of poly(butylene azelateco-butylene succinate) copolymers. Thermochim. Acta 2014, 575, 45-54. 
(14) Arandia, I.; Mugica, A.; Zubitur, M.; Arbe, A.; Liu, G.; Wang, D.; Mincheva, R.;

Dubois, P.; Müller, A. J. How Composition Determines the Properties of Isodimorphic Poly(butylene succinate-ran-butylene azelate) Random Biobased Copolymers: From Single to Double Crystalline Random Copolymers. Macromolecules 2015, 48, 43-57.

(15) Arandia, I.; Mugica, A.; Zubitur, M.; Iturrospe, A.; Arbe, A.; Liu, G.; Wang, D.; Mincheva, R.; Dubois, P.; Müller, A. J. Application of SSA thermal fractionation and Xray diffraction to elucidate comonomer inclusion or exclusion from the crystalline phases in poly(butylene succinate-ran-butylene azelate) random copolymers. J. Polym. Sci., Part B: Polym. Phys. 2016, 54, 2346-2358.

(16) Mincheva, R.; Delangre, A.; Raquez, J. M.; Narayan, R.; Dubois, P. Biobased Polyesters with Composition-Dependent Thermomechanical Properties: Synthesis and Characterization of Poly(butylene succinate-co-butylene azelate). Biomacromolecules 2013, 14, 890-899.

(17) Ye, H. M.; Wang, R. D.; Liu, J.; Xu, J.; Guo, B. H. Isomorphism in Poly (butylene succinate-co-butylene fumarate) and Its Application as Polymeric Nucleating Agent for Poly (butylene succinate). Macromolecules 2012, 45, 5667-5675.

(18) Allegra, G.; Bassi, I. W., Isomorphism in synthetic macromolecular systems. Adv. Polym. Sci. 1969, 6, 549-574.

(19) Pan, P.; Inoue, Y. Polymorphism and isomorphism in biodegradable polyesters. Prog. Polym. Sci. 2009, 34, 605-640.

(20) Schick, C.; Wurm, A.; Mohammed, A. Vitrification and Devitrification of the Rigid Amorphous Fraction in Semicrystalline Polymers Revealed from Frequency Dependent Heat Capacity. In Polymer Crystallization: Observations, Concepts and Interpretations, Reiter, G.; Sommer, J. U., Eds.; Springer: Berlin, Heidelberg, 2003; Vol. 14, p 252. 
(21) Coburn, J. C.; Boyd, R. H. Dielectric relaxation in poly(ethylene terephthalate). Macromolecules 1986, 19, 2238-2245.

(22) Alegría, A.; Colmenero, J. Dielectric relaxation of polymers: segmental dynamics under structural constraints. Soft Matter 2016, 12, 7709-7725.

(23) Kummali, M. M.; Alegría, A.; Miccio, L. A.; Colmenero, J. Study of the Dynamic Heterogeneity in Poly(ethylene-ran-vinyl acetate) Copolymer by Using Broadband Dielectric Spectroscopy and Electrostatic Force Microscopy. Macromolecules 2013, 46, $7502-7512$.

(24) Hill, A. J.; Tant, M. R., The Structure and Properties of Glassy Polymers. In Structure and Properties of Glassy Polymers, American Chemical Society, 1999; Vol. 710, p 1.

(25) Charlon, S.; Delbreilh, L.; Dargent, E.; Follain, N.; Soulestin, J.; Marais, S. Influence of crystallinity on the dielectric relaxations of poly(butylene succinate) and poly[(butylene succinate)-co-(butylene adipate)]. Eur. Polym. J. 2016, 84, 366-376.

(26) Tai, H. J. Dielectric spectroscopy of poly(butylene succinate) films. Polymer 2007, 48, $4558-4566$.

(27) Yu, L.; Ke, S.; Zhang, Y.; Shen, B.; Zhang, A.; Huang, H. Dielectric relaxations of high-k poly(butylene succinate) based all-organic nanocomposite films for Capacitor applications. J. Mater. Res. 2011, 26, 2493-2502.

(28) Starkweather, H. W.; Avakian, P.; Fontanella, J. J.; Wintersgill, M. C. Internal motions in polylactide and related polymers. Macromolecules 1993, 26, 5084-5087.

(29) Tatsumi, T.; Ito, E.; Hayakawa, R. Study of the dielectric $\beta$-relaxation in poly(ethylene terephthalate) and ethylene isophthalate terephthalate copolyesters. J. Polym. Sci., Part B: Polym. Phys. 1992, 30, 701-706. 
(30) Soccio, M.; Nogales, A.; Lotti, N.; Munari, A.; Ezquerra, T. A. The $\beta$ relaxation as a probe to follow real-time polymer crystallization in model aliphatic polyesters. Polymer 2007, 48, 4742-4750.

(31) Nogales, A.; Ezquerra, T. A.; García, J. M.; Baltá-Calleja, F. J. Structure-dynamics relationships of the $\alpha$-relaxation in flexible copolyesters during crystallization as revealed by real-time methods. J. Polym. Sci., Part B: Polym. Phys. 1999, 37, 37-49.

(32) Dantras, E.; Dandurand, J.; Lacabanne, C.; Caminade, A. M.; Majoral, J. P. TSC and Broadband Dielectric Spectroscopy Studies of the $\alpha$ Relaxation in Phosphorus-Containing Dendrimers. Macromolecules 2004, 37, 2812-2816.

(33) Gambino, T.; Martínez de Ilarduya, A.; Alegría, A.; Barroso-Bujans, F. Dielectric Relaxations in Poly(glycidyl phenyl ether): Effects of Microstructure and Cyclic Topology. Macromolecules 2016, 49, 1060-1069.

(34) Soccio, M.; Nogales, A.; Lotti, N.; Munari, A.; Ezquerra, T. A. Evidence of Early Stage Precursors of Polymer Crystals by Dielectric Spectroscopy. Phys. Rev. Lett. 2007, 98, 037801.

(35) Hofmann, A.; Alegría, A.; Colmenero, J.; Willner, L.; Buscaglia, E.; Hadjichristidis, N. Secondary and Segmental Relaxation in Polybutadienes of Varying Microstructure: Dielectric Relaxation Results. Macromolecules 1996, 29, 129-134.

(36) Shen, X.; Hu, W.; Russell, T. P. Measuring the Degree of Crystallinity in Semicrystalline Regioregular Poly(3-hexylthiophene). Macromolecules 2016, 49, 45014509 . 
For Table of Contents use only

\section{The Complex Amorphous Phase in Poly(butylene succinate-ran-butylene azelate) Isodimorphic Copolyesters}

Idoia Arandia ${ }^{1}$, Agurtzane Mugica ${ }^{1}$, Manuela Zubitur ${ }^{2}$, Rosica Mincheva ${ }^{3}$, Philippe Dubois ${ }^{3,4}$, Alejandro J. Müller ${ }^{* 1,5}$ and Angel Alegría ${ }^{* 6}$

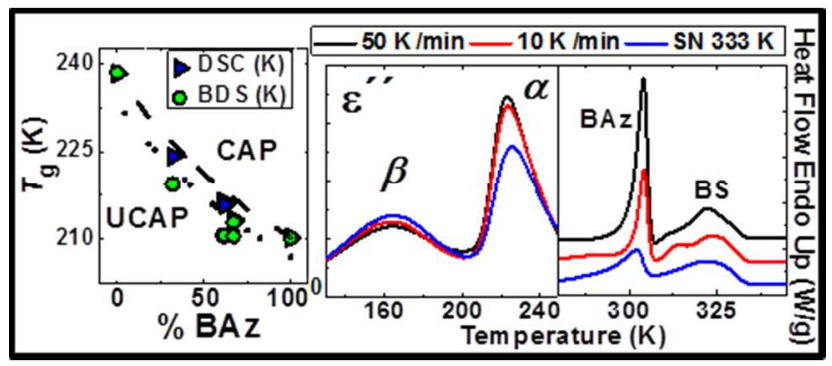

